\title{
Preparation of the Visible, Near Infrared and ShortWave Infrared detectors on EARTHCARE for the radiometric analysis of the earth energy balance
}

\author{
by J. Bentell ${ }^{1}$, K. van der Zanden ${ }^{1}$, U. Van Bogget ${ }^{1}$, T. Colin $^{1}$, P. Merken ${ }^{12}$, J. Vermeiren $^{1}$
}
(1) Xenics nv, Ambachtenlaan 44, BE-3001 Leuven, Belgium; http://www.xenics.com
(2) RMA, Renaissancelaan 30, BE-1000 Brussel, Belgium
(*) jan.vermeiren@xenics.com

\begin{abstract}
Earth care is a cornerstone mission of ESA to study the radiometric balance of the earth ecosystem. Whereas the influence of greenhouse gasses is well understood in the heat transfer kinetics, the role of clouds and aerosols are considerably less understood; they are nevertheless of very big importance for refining our knowledge of climate predictions and (local) weather forecasting. One of the instruments to measure the radiation budget is the Multi-Spectral Imager (MSI), giving a ground resolution at Nadir of $500 \mathrm{~m}$ and a $15 \mathrm{~km}$ wired swath and operating in 7 spectral bands ranging from the Red $(670 \mathrm{~nm})$ till the TIR $(12 \mathrm{?m})$. The Visible, NIR and SWIR (VNS) channels shall be mainly used for observing the return radiation on aerosols and clouds, while the TIR bands will be used to accurately measure the earth surface temperature. This paper will mainly focus on the requirements, design and qualification of the VNS channels for the MSI instrument. The response stability will be discussed in detail in order to fulfill the mission requirements
\end{abstract}

